\title{
Evolving
}

\section{Decellularized heart valve as a scaffold for in vivo recellularization: Deleterious effects of granulocyte colony-stimulating factor}

Francis Juthier, $\mathrm{MD}^{\mathrm{a}, \mathrm{b}}$ André Vincentelli, MD, PhD, ${ }^{\mathrm{a}, \mathrm{b}}$ Julien Gaudric, MD, ${ }^{\mathrm{a}, \mathrm{b}}$ Delphine Corseaux, PhD, Olivier Fouquet, MD, ${ }^{a, b}$ Christine Calet, MD, ${ }^{a, b}$ Thierry Le Tourneau, MD, PhD, ${ }^{a}$ Valérie Soenen, BS, ${ }^{c}$ Christophe Zawadzki, BS, ${ }^{a, c}$ Olivier Fabre, MD, ${ }^{a, b}$ Sophie Susen, MD, ${ }^{a, c}$ Alain Prat, MD, ${ }^{a, b}$ and Brigitte Jude, MD, PhD ${ }^{a, c}$

From Institut National de la Santé et de la Recherche Médicale (Inserm) ERI-9, Faculté de Médecine, ${ }^{a}$ Lille, France, the Centre Hospitalier Régional Universitaire de Lille, Clinique de Chirurgie Cardiovasculaire, Lille, France, and Institut d'HématologieTransfusion, ${ }^{c}$ Lille, France

Supported in part by grants from the French Fond National pour la Science, Ministère de la Recherche, Action Concertée Incitative 2002-Grant 02TS 050 and from Conseil Régional Nord Pas de Calais 2003OBJ2-2004/1-4-1, no. 157.

Received for publication Aug 5, 2005; revisions received Nov 20, 2005; accepted for publication Nov 28, 2005.

Address for reprints: André Vincentelli, $\mathrm{MD}, \mathrm{PhD}$, Clinique de Chirurgie Cardiovasculaire, Hôpital Cardiologique, 59037 Lille cedex (E-mail: a-vincentelli@ chru-lille.fr).

J Thorac Cardiovasc Surg 2006;131:843-52 0022-5223/ $\$ 32.00$

Copyright $\odot 2006$ by The American Association for Thoracic Surgery

doi:10.1016/j.jtcvs.2005.11.037
Background: Autologous recellularization of decellularized heart valve scaffolds is a promising challenge in the field of tissue-engineered heart valves and could be boosted by bone marrow progenitor cell mobilization. The aim of this study was to examine the spontaneous in vivo recolonization potential of xenogeneic decellularized heart valves in a lamb model and the effects of granulocyte colony-stimulating factor mobilization of bone marrow cells on this process.

Methods: Decellularized porcine aortic valves were implanted in 12 lambs. Six lambs received granulocyte colony-stimulating factor $\left(10 \mu \mathrm{g} \cdot \mathrm{kg}^{-1} \cdot \mathrm{d}^{-1}\right.$ for 7 days, granulocyte colony-stimulating factor group), and 6 received no granulocyte colony-stimulating factor (control group). Additionally, nondecellularized porcine valves were implanted in 5 lambs (xenograft group). Angiographic and histologic evaluation was performed at $3,6,8$, and 16 weeks.

Results: Few macroscopic modifications of leaflets and the aortic wall were observed in the control group, whereas progressive shrinkage and thickening of the leaflets appeared in the granulocyte colony-stimulating factor and xenograft groups. In the 3 groups progressive ovine cell infiltration (fluorescence in situ hybridization) was observed in the leaflets and in the adventitia and the intima of the aortic wall but not in the media. Neointimal proliferation of $\alpha$-actin-positive cells, inflammatory infiltration, adventitial neovascularization, and calcifications were more important in the xenograft and the granulocyte colony-stimulating factor groups than in the control group. Continuous re-endothelialization appeared only in the control group.

Conclusion: Decellularized xenogeneic heart valve scaffolds allowed partial autologous recellularization. Granulocyte colony-stimulating factor led to accelerated heart valve deterioration similar to that observed in nondecellularized xenogeneic cardiac bioprostheses.

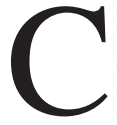
linically available cardiac valve prostheses have long-term limitations, including the need for lifelong anticoagulation for mechanical prosthesis and limited durability for bioprostheses. ${ }^{1}$ Tissue engineering could create an ideal valve, with physiologic hemodynamic behavior, low immunogenic potential, 


\section{Abbreviations and Acronyms \\ $\mathrm{BM}=$ bone marrow \\ $\mathrm{G}-\mathrm{CSF}=$ granulocyte colony-stimulating factor \\ $\mathrm{VWF}=$ Von Willebrand Factor}

and repair, remodeling, and growing capabilities. Several groups demonstrated the feasibility of creating living valves in vitro through seeding autologous cells on various scaffolds, such as synthetic polymers, collagen, and allogeneic or xenogeneic valve conduits. ${ }^{2-4}$ Xenogeneic natural scaffolds have the advantage of adequate anatomic structure and unlimited availability. However, they can trigger deleterious immune responses, especially cell-mediated ones. Decellularization of xenogeneic valve matrices reduces their antigenicity, and recent in vitro studies suggest that decellularized porcine valve matrices are a suitable scaffold for recolonization by smooth muscle cells. ${ }^{5}$

The strategy to promote the recolonizing process remains questionable. First experiments were conducted with differentiated vascular cells (endothelial cells or smooth muscle cells) obtained from samples of saphenous veins or autologous carotid arteries. ${ }^{2,6}$ More recently, bone marrow (BM) cells have been demonstrated to contain various progenitor cells, such as endothelial progenitors and mesenchymal lineage cells, which can induce tissue regeneration when injected into cardiovascular structures. ${ }^{7}$ Cardiac valve interstitial cells are myofibroblasts of mesenchymal origin, and it was demonstrated that BM cells could recolonize synthetic valve scaffolds in vitro, thus confirming that BM cells contain proper cell progenitors for heart valve reconstruction. ${ }^{8,9}$

Early BM progenitor cells can be mobilized by growth factors, such as granulocyte colony-stimulating factor (G-CSF), into peripheral blood. Numerous experimental studies have shown that G-CSF-mobilized BM cells are able to home to injured cardiovascular tissues and contribute to tissue regeneration, especially in infarcted myocardium and hind-limb ischemic areas. ${ }^{10,11}$

The aim of this study was to examine the spontaneous in vivo recolonization potential of xenogeneic decellularized heart valves in a lamb model and the effects of G-CSF mobilization of BM cells on this process.

\section{Methods \\ Sampling and Decellularization of Porcine \\ Aortic Valves}

Aortic valve conduits were sampled from pigs (Large white/ Landras, $10-15 \mathrm{~kg}$ of body weight). The animals were anesthetized by means of intravenous injection of propofol, $20 \mathrm{mg} / \mathrm{kg}$, and sufentanil, $1 \mu \mathrm{g} / \mathrm{kg}$. After median sternotomy, the heart was explanted under surgical conditions. Aortic valve conduits were harvested with a thin ridge of subvalvular muscle tissue proximally and a short arterial segment distally. Then porcine valve conduits were calibrated with a Hegar dilatator, weighed, and washed in Hank's balanced salt solution plus aprotinin (10 KIU/mL), penicillin $(100 \mathrm{U} / \mathrm{mL})$, streptomycin $(100 \mu \mathrm{g} / \mathrm{mL})$, and nystatin (100 U/mL) plus N-2-hydroxyethylpiperazine-N-2-ethanesulfonic acid $(10 \mathrm{mmol} / \mathrm{L})$ at $\mathrm{pH}$ 7.6. Valve conduits were decellularized through a nonenzymatic procedure, associating hypotonic shock and low-concentration ionic detergent, as previously described. ${ }^{12,13}$ Briefly, valve conduits were incubated at $20^{\circ} \mathrm{C}$ during constant stirring in hypotonic buffer (Tris, $10 \mathrm{mmol} / \mathrm{L}$; ethylendiamine tetraacetic acid, 0.1\%; and aprotinin, $10 \mathrm{KIU} / \mathrm{mL}$; $\mathrm{pH} 8$ ) for 14 hours and hypotonic buffer with sodium dodecylsulfate $(0.1 \%)$ for 24 hours, washed in isotonic buffer (Tris, $50 \mathrm{mmol} / \mathrm{L} ; \mathrm{NaCl}, 0.15$ $\mathrm{mol} / \mathrm{L}$; ethylendiamine tetraacetic acid, $0.1 \%$; aprotinin, $10 \mathrm{KIU} /$ $\mathrm{mL} ; \mathrm{pH} 8$ ) for 24 hours, and then immediately implanted in lambs. Moreover, additional porcine valve conduits were sampled and stored in Hank's balanced salt solution plus aprotinin (10 KIU/mL), penicillin $(100 \mathrm{U} / \mathrm{mL})$, streptomycin $(100 \mu \mathrm{g} / \mathrm{mL})$, and nystatin (100 U/mL) plus N-2-hydroxyethylpiperazine-N-2-ethanesulfonic acid $(10 \mathrm{mmol} / \mathrm{L})$ at $\mathrm{pH} 7.6$ for 72 hours at room temperature without any decellularization process. In all valves sterility controls were realized on the last washing solution.

\section{Study Design}

In 17 lambs (Romanov/Ile de France; aged 12-16 weeks; median weight, $23 \mathrm{~kg}$ ) a porcine aortic valve conduit was implanted in the descending thoracic aorta. Five lambs received a nondecellularized porcine valve (xenograft group). Twelve lambs received a decellularized porcine valve. Among them, 6 received subcutaneous injections of G-CSF (Neupogen, a kind gift from Amgen Laboratories) (10 $\mu \mathrm{g} / \mathrm{kg}$ for 7 days, from day 3 before to day 3 after the operation; G-CSF group), and 6 received no G-CSF (control group).

\section{Surgical Techniques}

In all animals general anesthesia was induced and maintained by means of intravenous injection of propofol, $20 \mathrm{mg} / \mathrm{kg}$, and sufentanil, $1 \mu \mathrm{g} / \mathrm{kg}$. All animals were operated on by the same team of surgeons (AV and FJ). The descending aorta was exposed through a left anterolateral thoracotomy, entering the chest through the fourth intercostal space. The aorta was dissected and exposed at the level of the isthmus. Two 4-0 monofilament purse strings were made on the aortic arch and distally on the descending aorta (Prolene, Ethicon, Inc). Systemic anticoagulation was induced with heparin (200 UI/kg), and a 14-mm diameter pediatric gastric tube was implanted between the aortic arch and the thoracic descending aorta as an aorticoaortic shunt. Then the aorta was crossclamped proximally immediately after the origin of the left subclavian artery and 15 $\mathrm{cm}$ distally. The descending aorta was transected, and the porcine aortic valve conduit was inserted with 2 end-to-end 4-0 monofilament running sutures. On completion of the operation, heparin was reversed with protamine (200 UI/kg). The chest was closed in layers, and a chest tube was inserted. The chest tube was removed after extubation. Animals were then settled in standard conditions, with food and drink ad libitum. All animals received $1000 \mathrm{mg}$ of ceftriaxon and $500 \mathrm{mg}$ of aspirin for the first postoperative week on a daily basis. For pain control, during the first 2 operative days, a transdermal fentanyl patch 

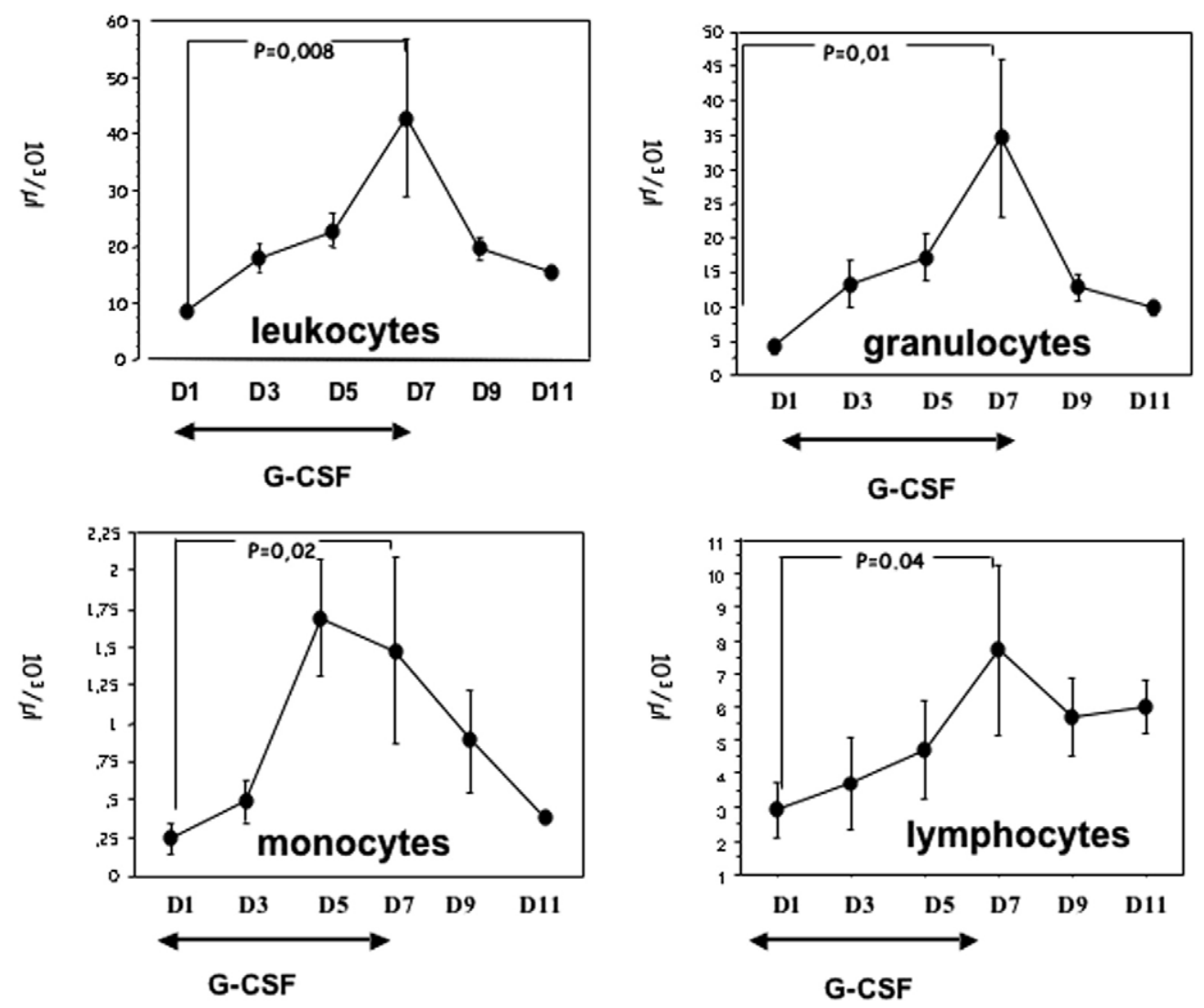

Figure 1. Kinetics of white blood cell counts in lambs after subcutaneous injection of recombinant human G-CSF (10 $\mu \mathrm{g} / \mathrm{kg}$ ) for 7 days.

was applied on the chest. All the animals received humane care in compliance with the "Guide for the Care and Use of Laboratory Animals" published by the National Institutes of Health (National Institutes of Health publication no. 85-23, revised 1985).

\section{Valve Follow-up and Explantation Process}

In each group euthanasia was performed in one lamb at 3,6, and 8 weeks and in the remaining animals at 16 weeks after implantation. Euthanasia was performed under the same protocol of analgesia and anesthetic procedures as for implantation. Before termination, heparin (300 UI/kg) was administrated, and an aortography was realized in each animal after catheterization of the femoral artery to detect graft abnormalities (thrombosis, aneurysm, and stenosis) and to measure the transvalvular gradient. Grafts were explanted together with the descending thoracic aorta.

\section{Valve Analysis}

Explanted valve conduits were grossly examined. Aneurysmal aortic dilatation, thickness, and retraction of the cusps and the presence of visible calcifications were reported. Then fragments from the grafted aortic wall and cusps were sampled for cytologic analysis of colonizing cells and histologic analysis.
For histology, samples were fixed in a buffered $4 \%$ formaldehyde solution, dehydrated, and embedded in paraffin. Sections $(6 \mu \mathrm{m})$ were stained with hematoxylin, eosin, and safran and with Masson trichrome. For analysis of the intima/media ratio, computed planimetry was realized through Perfect Image 7.10 software (Clara Vision). In addition, orcein staining for elastic fibers and Von Kossa staining for calcium were performed.

Immunostaining of the paraffin sections was done with monoclonal antibody to $\alpha$-actin $\left(1: 20,1\right.$ night at $\left.+4^{\circ} \mathrm{C}\right)$ or with polyclonal antibody to VWF $(1: 500,2$ hours at room temperature; DAKO) and respective isotope-matched IgG control (Cymbus Biotechnology). The immunoreaction was detected with alkaline phosphatase for $\alpha$-actin and with Avidin Biotin performed Complex peroxidase for VWF. The tissue sections were analyzed by 2 independent observers (DC and BJ) who were blinded to the animal group allocation.

\section{Cytologic Analysis and Molecular Probes}

For cell isolation, tissue samples were digested for 45 minutes at $37^{\circ} \mathrm{C}$ in a solution containing type I collagenase, elastase, and soybean trypsin inhibitor. After addition of $30 \%$ fetal calf serum, cytocentrifuged preparations were made and frozen at $-70^{\circ} \mathrm{C}$. Fluorescence in situ hybridization was performed with a bacterial artificial chromosome (BAC 31EA; a kind gift of Dr François 


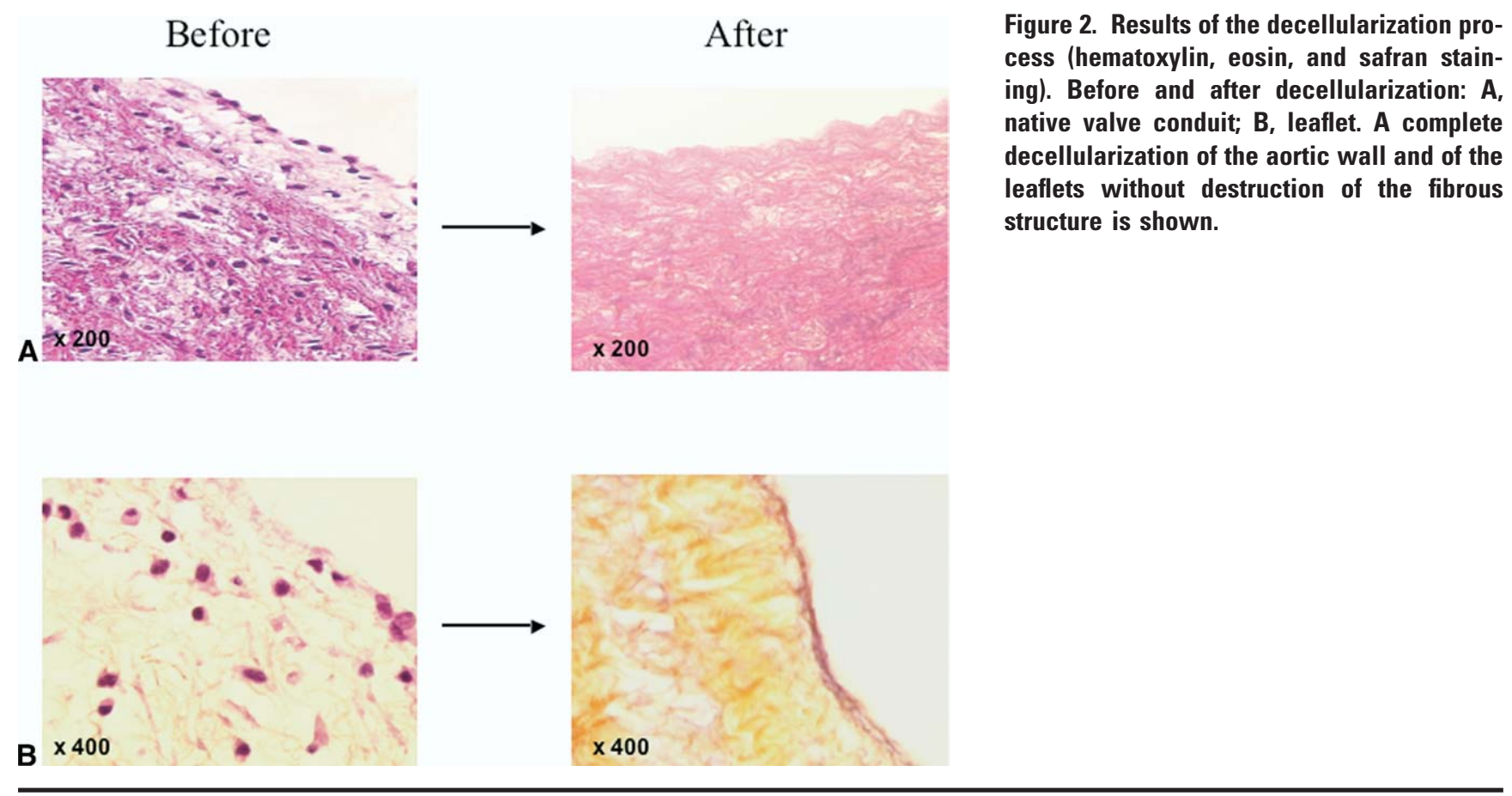

Piumi, Institut National de Recherche Agronomique, Jouy en Josas, France) to detect the presence of cells of ovine origin in the samples. The ovine pancentromeric probe was labeled in red by means of nick-translation (Spectrum Red dUTP and Nick Translation Reagent kit, Vysis Inc). The slides were fixed, treated with mild pepsin solution, and dehydrated. After denaturation, identification of the centromers was performed by means pf overnight hybridization at $37^{\circ} \mathrm{C}$. After washing, DNA was counterstained in blue with 4',6 diamino-2-phenylindole. A red labeling of the chromosomes was observed in all ovine cells, and no labeling was observed in porcine cells.

\section{Statistical Analysis}

Statistical analysis was performed with Statview software (SAS Institute Inc). Continuous variables were expressed as means \pm standard deviations. The Wilcoxon test was used to compare individual blood cell values over the time course of the experiments. The Kruskall-Wallis test was used to analyze differences in the intima/media ratio among the 3 groups.

\section{Results}

\section{Effects of G-CSF on Lamb White Blood Cell Counts}

Because the effects of recombinant human G-CSF in lambs had not been reported yet, we first tested the kinetics of peripheral white blood cell counts after G-CSF administration in 4 lambs. A daily subcutaneous injection of $10 \mu \mathrm{g} / \mathrm{kg}$ G-CSF was performed for 7 days, and blood sampling was performed every 2 days. An increase in all white cell counts was observed as soon as 2 days after injection and was maximal at day 7 (Figure 1). The mean counts of leukocytes, granulocytes, monocytes, and lymphocytes at day 7 exceeded baseline values by 5-, 8.6-, 4.6-, and 2.6-fold, respectively $(P \leq .04)$.

\section{Results of the Decellularization Process on Aortic Valve Conduits}

The decellularization process did not induce macroscopic modifications of the valve conduits. Histologic analysis showed no remaining cells in the cusps and rare pyknotic nucleus in the deepest part of the media and in the boarding myocardial tissue. The organization of collagen fibers was similar to that of a native valve conduit (Figure 2). Valve conduits were equally decellularized in the control group and in the G-CSF group before implantation.

\section{Valve Follow-up and Outcome}

Angiography showed no aneurysmal dilatation or stenosis at any time in all 3 groups, except in one animal in the xenograft group that exhibited valve thrombosis at 6 weeks (Figure 3 ). In all other cases, no hemodynamic abnormality was observed. In particular, no transvalvular gradient or systemic hypertension was observed in any animal. In the xenograft and the G-CSF groups, gross observation of the valves showed calcifications of the leaflets and of the aortic wall, leaflet thickening, and shrinkage at 6,8 , and 16 weeks. None of these lesions were observed in the decellularized group (Figure 4).

\section{Histologic and Cytologic Analysis}

Representative histologic features of the 3 groups at 16 weeks are shown in Figures 5 and 6 (top row, control group; 

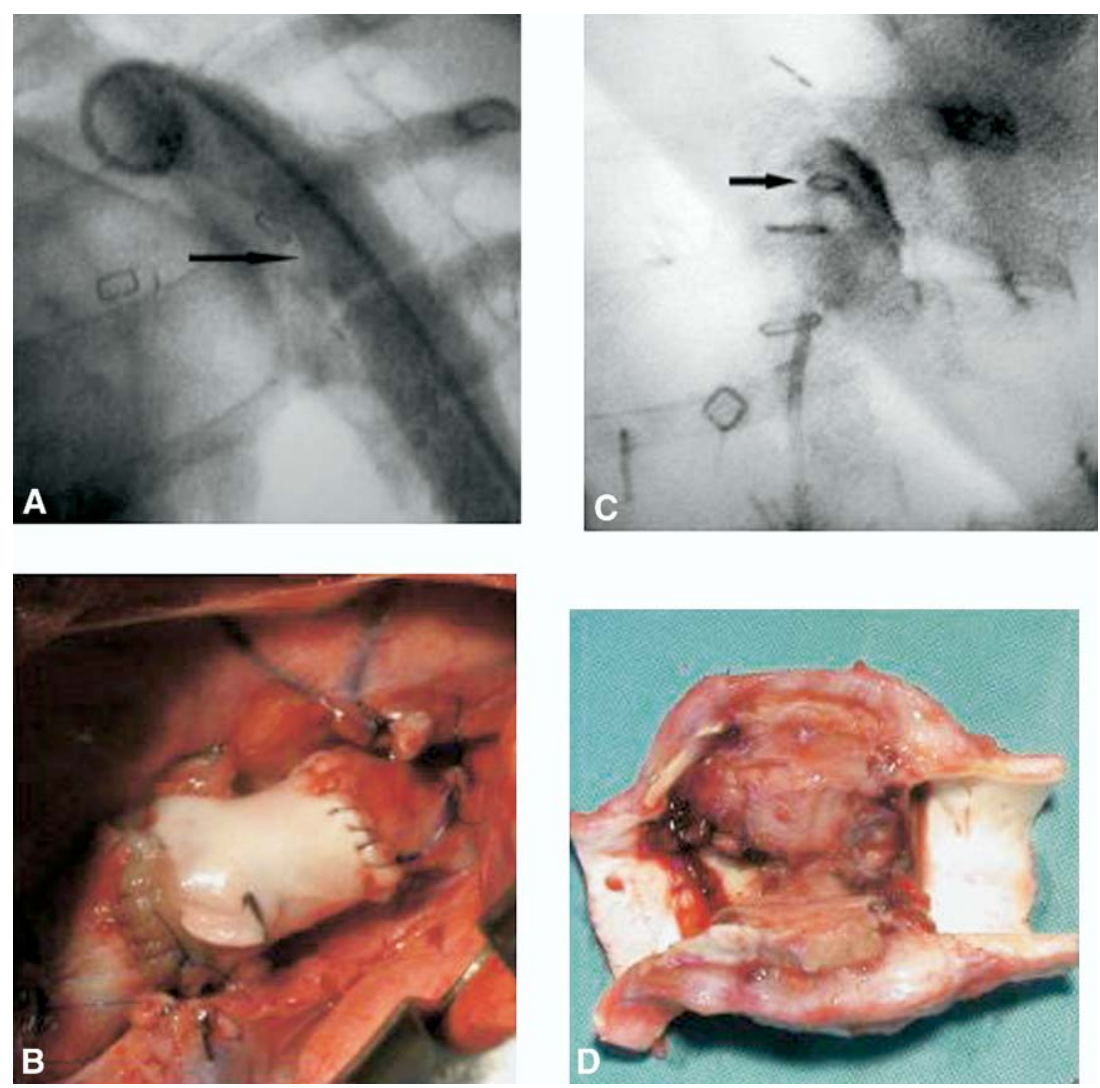

Figure 3. A and B, Representative aspects of normal aortography (A) at 16 weeks and perioperative aspect of the graft in the thoracic descending aorta (B). C and D, Specific aspect of the 16 weeks after implantation aortography (C) and of the explanted valve (D) in the animal that underwent complete valve thrombosis (xenograft group). Arrows indicate site of the graft implantation.

middle row, G-CSF group; and bottom row, xenograft group). In the control group inflammatory cell infiltration and few small vessels were observed in the adventitia after 3 weeks but remained limited and did not increase during follow-up (Figure 5, $A$ and $B$ ). A progressive neointimal thickening appeared after 6 weeks and increased up to 16 weeks (Figure 5, $C$ ). $\alpha$-Actin staining showed numerous positive cells in the neointima (Figure $5, D$ ). No cell was visible in the media at any time. No modification of the elastic fibers was observed (orcein staining; Figure 6, A). Rare microcalcifications appeared after 16 weeks in the junction between adventitia and media (Von Kossa staining; Figure 6, B). A continuous endothelial layer was seen on the luminal surface after 6 weeks and up to 16 weeks on the aortic wall and the leaflets (VWF staining; Figure 6,C). Few cells were observed in the leaflets (Figure 6,D), some of them being positive for $\alpha$-actin staining.

In the G-CSF group a progressive inflammatory infiltration appeared in the adventitia at 3 weeks, which increased with time and was associated with the development of numerous neovessels at 16 weeks (Figure 5, $A$ and $B$ ) and of a marked neointimal proliferation of $\alpha$-actin-positive cells (Figure 5, $C$ and $D$ ). Necrotic zones were visible in the media, which remained acellular. Elastic fibers exhibited a progressive disorganization, thickening, and fragmentation (orcein staining; Figure 6, A). Von Kossa staining showed microcalcifications in the aortic wall and in the leaflets after 6 weeks. Elastic fiber disorganization and heavy calcifications at the adventitia-media and the media-neointima junctions were observed (Figure 6, B). VWF staining showed only a discontinuous endothelial cell layer on the luminal surface (Figure 6,C). Necrosis and inflammatory infiltration was also visible in the leaflets associated with calcifications (Figure 6, D).

In the xenograft group a marked inflammatory infiltration and neovascularization was observed in the adventitia as soon as after 3 weeks and increased up to 16 weeks (Figure 5, $A$ and $B$ ). Calcifications were visible as soon as 6 weeks. At 16 weeks, the aortic wall and leaflets exhibited features similar to those of the G-CSF group, with marked neointimal thickening (Figure 5, $C$ and $D$ ), disorganization of elastic fibers (Figure 6, A), calcifications both in the 

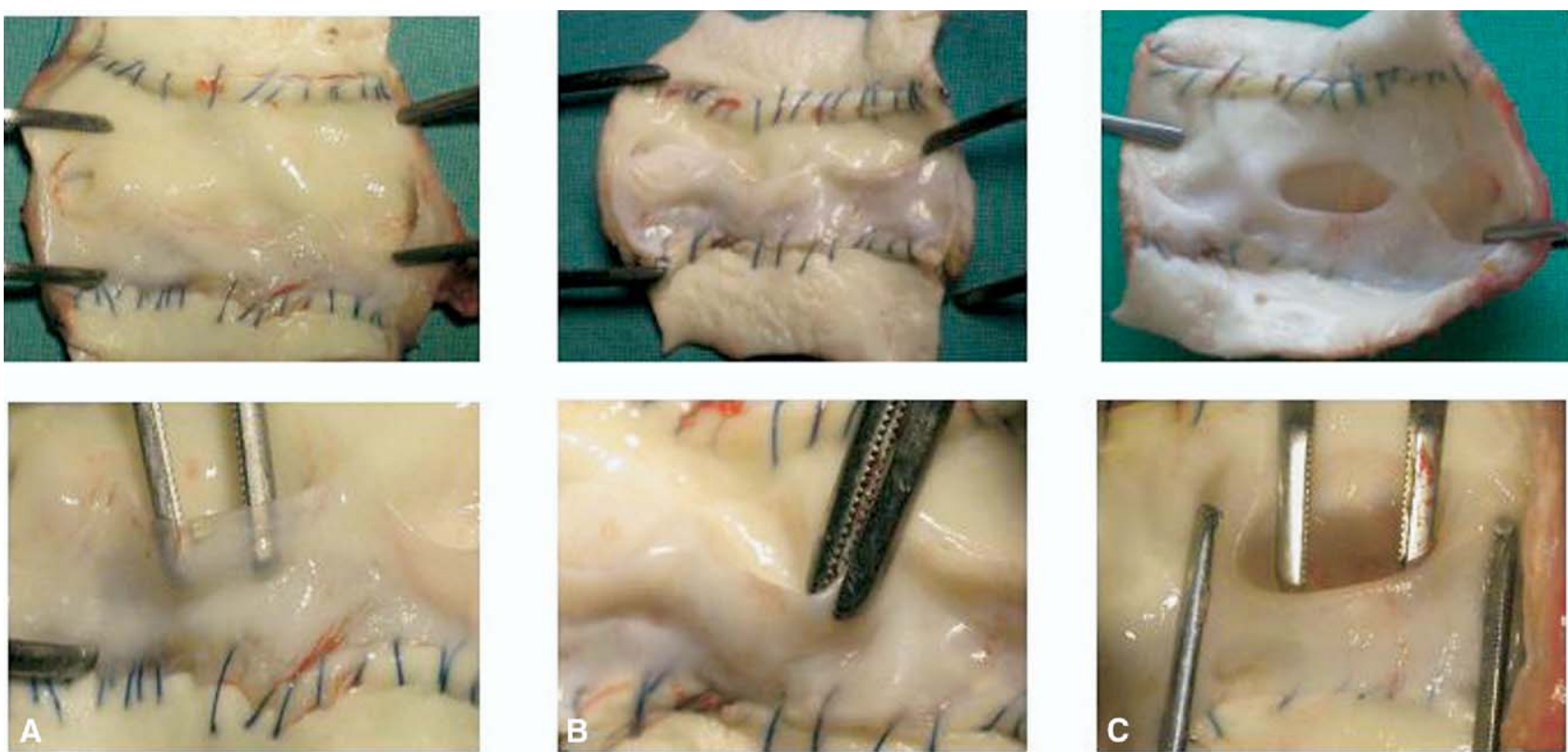

Figure 4. Macroscopic examination at 16 weeks. A, Control group; B, G-CSF group; C, Xenograft group. In the xenograft and the G-CSF groups, but not in the control group, gross observation showed calcifications of the leaflets and of the aortic wall, leaflet thickening, and shrinkage at 16 weeks.

aortic wall and the leaflets (Figure 6, $B$ and $D$ ), and discontinuous endothelialization (Figure 6, $C$ ).

Comparison of the neointima/media thickness showed that the intima/media ratio increased continuously with time in the 3 groups. This increase was more rapid and pronounced in the xenograft group and the G-CSF group than in the decellularized group at 3 weeks (Figure 7). However, at 16 weeks, the intima/media ratio tended to be the same in the 3 groups.

All the cells eluted from the grafted decellularized valves were stained by the centromeric probe, demonstrating their autologous ovine origin (Figure 8).

\section{Discussion}

In this study decellularized porcine valve scaffolds implanted in juvenile sheep exhibited good mechanical resistance under high systemic strain after 16 weeks and were partially recolonized by ovine cells in the leaflets and the aortic wall. The healing process led to the formation of a neointimal proliferation generated by ovine smooth muscle cells. Treatment with G-CSF did not improve scaffold recolonization but rather induced accelerated deterioration of the valve, which was similar to the xenograft rejection process observed in nondecellularized porcine valves.

As a biologic extracellular matrix scaffold, we chose porcine heart valves for their well-known good hemodynamic behavior and unlimited availability. When used as valve prostheses, these porcine scaffolds are usually treated with glutaraldehyde to improve mechanical properties and to limit the xenogeneic rejection process. However, glutaraldehyde treatment profoundly modifies the extracellular matrix structure and makes it improper to support cell migration, recolonization, and the matrix-renewing process. ${ }^{14} \mathrm{De}$ cellularization of porcine valves is another approach to limit cell-mediated xenograft rejection and potentially facilitate recolonization by interstitial cells. Several decellularization processes have been described, leading to important differences in the efficiency of cell removal, long-term mechanical properties, and xenoantigen residual exposition. ${ }^{15,16} \mathrm{It}$ is noteworthy that in a series of 4 young patients, enzymatic decellularization of porcine grafts have shown severe drawbacks associated with catastrophic clinical results, such as rapid graft failure, that were responsible for the death of two thirds of the patients. ${ }^{17,18}$ By contrast, homografts decellularized with the same enzymatic process (Synergraft, Cryolife Inc) and implanted in adults have shown better outcome. ${ }^{19}$ Furthermore, the postmortem analysis of a Synergraft-treated homograft explanted 5 weeks after implantation in a 60-year-old patient showed preserved morphology, although associated with inflammatory infiltration. ${ }^{20}$ In our study we used porcine xenografts decellularized through a nonenzymatic process on the basis of osmotic shock, detergent cell extraction ( $0.1 \%$ sodium dodecylsulfate), and antiproteases recently described by Korossis and colleagues, ${ }^{12}$ which induced complete decellularization without major impairment of the structural proteins. Under that process, valve conduit tissues had equal strength compared with fresh tissues and only modest changes in extensibility 

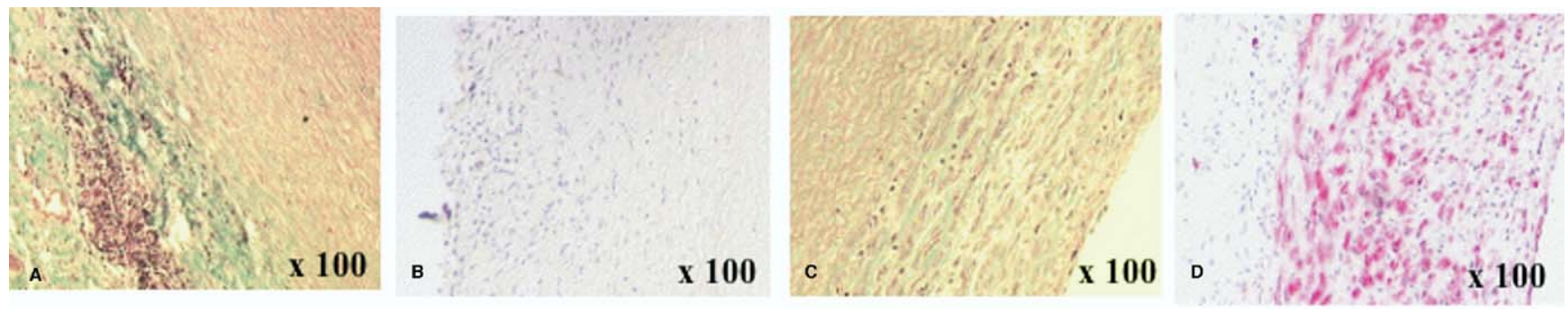

\section{Control group}
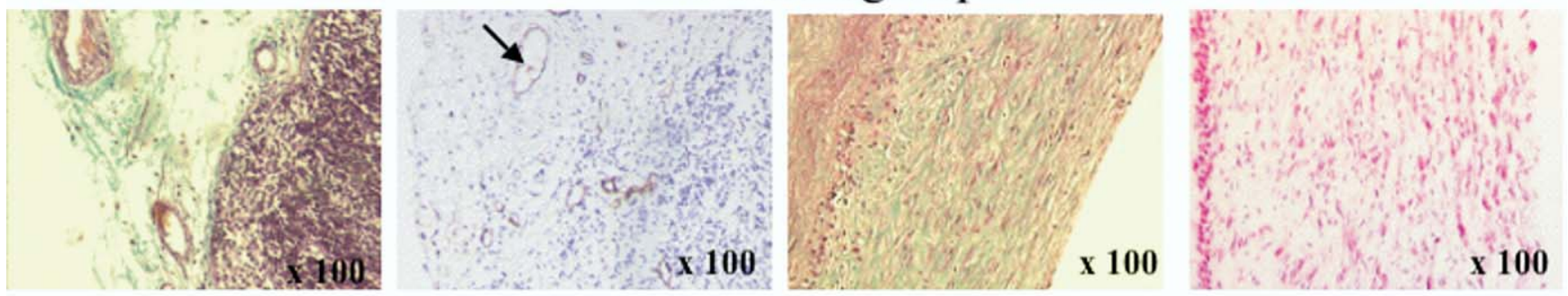

\section{G-CSF group}
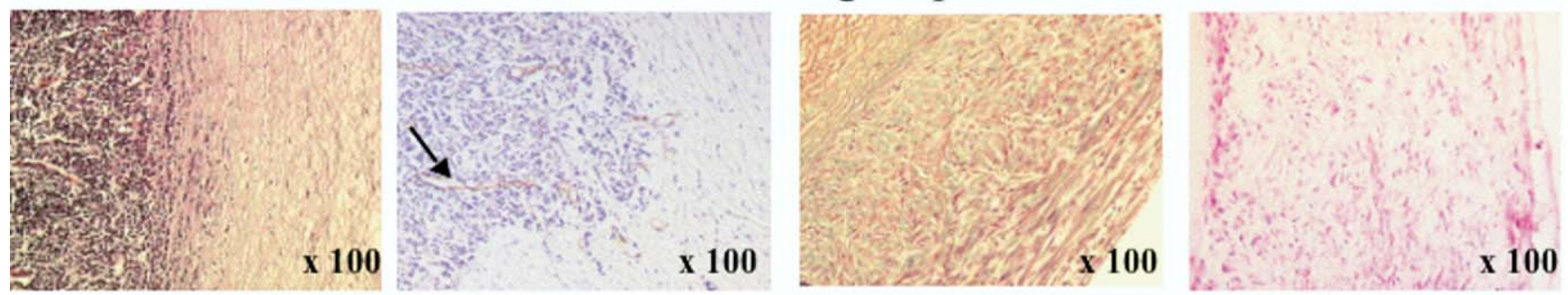

\section{Xenograft group}

Figure 5. Representative histologic aspects in the grafted aortic wall at $\mathbf{1 6}$ weeks in the $\mathbf{3}$ groups (top row, control group; middle row, G-CSF group; bottom row, xenograft group). A, Adventitia (Masson trichrome staining): collagen fibers and limited infiltration by inflammatory cells were visible in the control group. Inflammatory cells were more prominent in the G-CSF and xenograft groups than in the control group. B (anti-VWF antibody), Adventitial neovascularization (arrows) was limited in the control group and abundant in the G-CSF and xenograft groups. C (Masson trichrome staining), Neointimal proliferation tended to be more important in the G-CSF and xenograft groups than in the control group. D (anti- $\alpha$-actin staining), Evidence of strong $\alpha$-actin staining in the neointimal layer in the 3 groups.

in vitro. ${ }^{13}$ In addition, this process was recently demonstrated to remove xenoantigens. ${ }^{21}$ Using that protocol, we observed a complete tissue decellularization before implantation, and this observation was further confirmed after explantation because all the cells eluted from the explanted valves were positive for the ovine centromeric probe, demonstrating their ovine origin.

Such decellularized scaffolds were recently demonstrated in vitro to allow cell recolonization by smooth muscle cells without cytotoxicity..$^{5}$ Our study analyzes for the first time the ability of this decellularized scaffold to be recolonized in vivo by nonporcine cells. Because high mechanical forces must be sustained by the aortic valve conduit, we implanted the scaffolds in the descending aorta of lambs to evaluate its resistance to physiologic strains in vivo. As previously reported by Korossis and colleagues ${ }^{12}$ in vitro, we observed that these scaffolds have a good mechanical resistance in vivo, with no aneurysm formation and no rupture. We chose, in this study, to implant the porcine scaffolds in the descending aorta of lambs, where systemic strains are very high. Such an implantation site allows us to test the aortic root resistance to high mechanical strains but does not allow evaluation of the aortic leaflet functions.

We observed a limited recolonization associated with weak inflammatory reaction, no visible calcifications, and delayed neointimal proliferation, suggesting that this scaffold could be suitable for in vivo homing. Whether these 

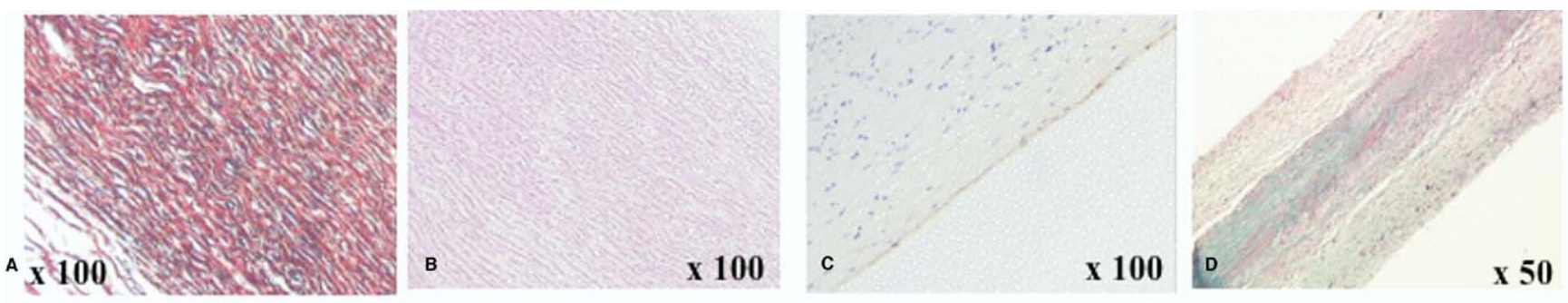

\section{Control group}
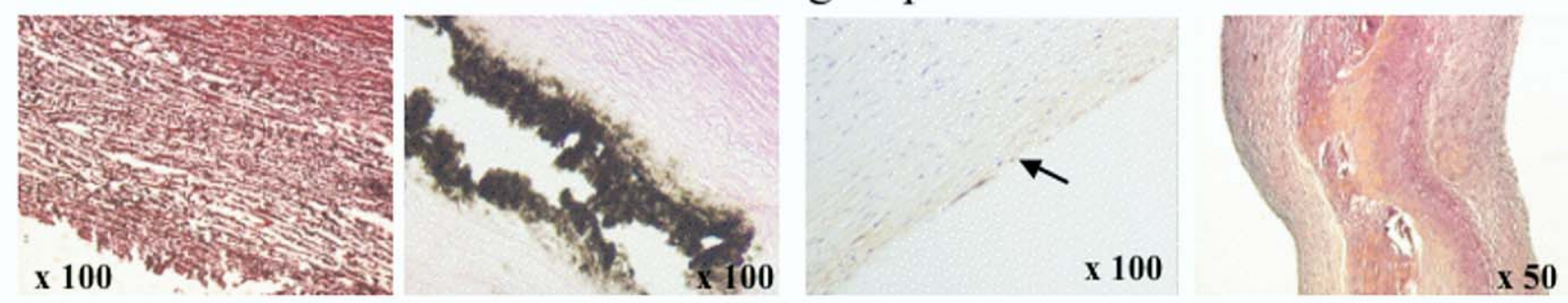

\section{G-CSF group}
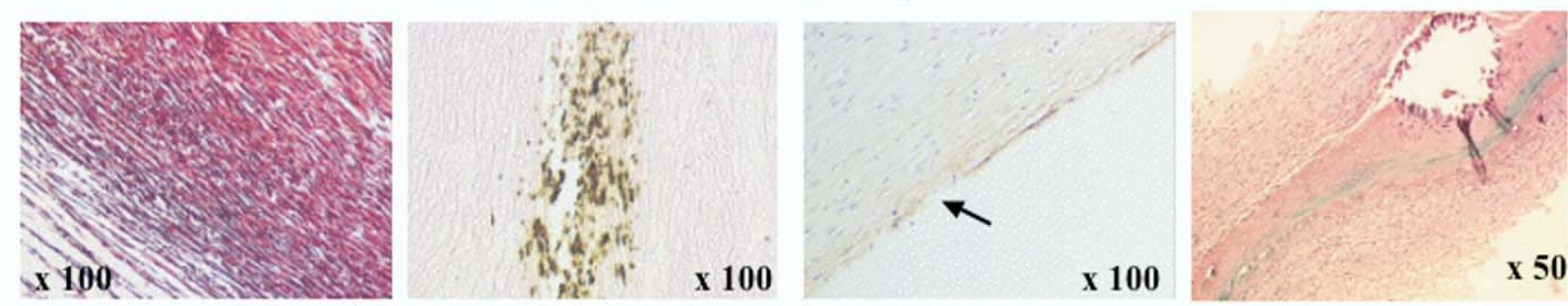

\section{Xenograft group}

Figure 6. Orcein, von Kossa, and immunochemical staining with anti-VWF antibody in the 3 groups and representative aspects of the leaflets are shown (top row, control group; middle row, G-CSF group; bottom row, xenograft group). A (orcein staining), Elastic fibers remained parallel in the control group and were disrupted and disorganized in the xenograft and G-CSF groups. B (Von Kossa staining), Evidence of heavy calcifications in the aortic wall in the G-CSF and xenograft groups and not in the control group. C (anti-VWF antibody), The luminal surface of the aortic wall was covered by a continuous layer of endothelial cells in the control group and by a disrupted layer (arrows) in the G-CSF and xenograft groups. D (Masson trichrome staining), Representative aspects of the leaflets in the 3 groups, with calcifications in the G-CSF and xenograft groups.

cells can produce collagen to repair the valve remains to be investigated. Moreover, the media of the aortic wall remained acellular, and although delayed, neointimal proliferation was comparable with that of nondecellularized grafts after 16 weeks.

In an attempt to optimize scaffold recolonization, a group of lambs received G-CSF, a growth factor routinely used in human clinical applications, which can mobilize multipotent progenitor cells from BM into peripheral blood. In myocardial infarction beneficial effects of G-CSF, such as decreased left ventricular remodeling and improved myocardial function, have been obtained in animal models. ${ }^{22-24}$ The advocated mechanisms for these beneficial effects are improvement of the postinfarct healing process through increased macrophage infiltration, promotion of reparative collagen synthesis in the infarct area, inhibition of apoptosis, and increased neovascularization. ${ }^{25} \mathrm{G}-\mathrm{CSF}$ has also been demonstrated to improve endothelialization of vascular grafts in dogs, ${ }^{26}$ suggesting that it could mobilize early progenitors for endothelial cells. Moreover, it was recently demonstrated that G-CSF mobilizes functional endothelial progenitor cells in patients with coronary disease. ${ }^{27}$

Because human G-CSF had not been tested previously in lambs, we first verified that it could induce a significant increase in peripheral white blood cell counts in our model. The observed increase was significant, although less pronounced than in human subjects. ${ }^{28}$ Unfortunately, immuno- 


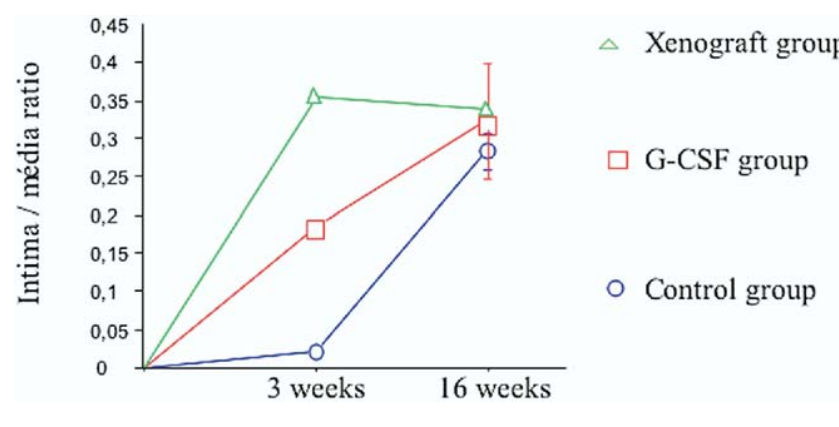

Figure 7. Time course of the intima/media ratio in the 3 groups.

logic identification of stem cells was not possible in this model because available human antibodies against human or mouse stem cell antigens do not react with sheep stem cells. In grafted decellularized heart valves, G-CSF induced an increase in inflammatory cell infiltration and neovessel formation in the adventitia, myointimal proliferation, and calcifications, both in leaflets and the aortic wall, indicating deleterious effects on valve outcome.

Several mechanisms could account for these observations. G-CSF is a physiologic component of the acutephase response, and its primary effect is the mobilization of neutrophil granulocytes. ${ }^{29} \mathrm{G}-\mathrm{CSF}$-mobilized cells also contain a significantly higher proportion of monocytes than normal peripheral blood and of accessory cells, particularly T-cell subsets. Therefore G-CSF can mobilize various subsets of mature effector cells of the immune and inflammatory response, which could amplify the immune and inflammatory reaction against residual extracellular matrix antigens of the decellularized scaffold. Although the decellularization process we used has been shown to be the most effective on xenoantigen removal, ${ }^{21}$ recent data indicate that porcine scaffolds decellularized with a similar method keep a significant potential to attract monocytes and promote their migration in extracellular matrix. ${ }^{16}$ Therefore the deleterious effects of G-CSF could be related to a dramatic increase of leukocyte-mediated immune and inflammatory reaction against residual xenoantigens, as observed in nondecellularized xenografts. Decellularized allogeneic valves could provide more adequate scaffolds for autologous recellularization, as recently demonstrated. ${ }^{16}$ The effects of G-CSF on such allogeneic scaffolds remain to be investigated.

It is noteworthy that the deleterious effects of G-CSF have recently been described in a clinical assay in myocardial infarction in human subjects, in whom G-CSF treatment resulted in increased restenosis rates after coronary stenting. ${ }^{30} \mathrm{We}$ also observed an increased myointimal proliferation in the grafted aortic wall, as well as increased neovessel formation in the adventitia, indicating that G-CSF induces significant and potentially deleterious effects in the aortic wall remodeling process. Although we did not observe any aneurysmal formation, such an early inflammatory reaction could lead to significant weakening of the valve prosthesis and to accelerated valve failure.

The immature sheep model has been used for years for testing bioprosthetic tissue, which degenerates rapidly, as in young patients, with morphology similar to that seen in clinical specimen. Although porcine valve scaffolds decellularized through a nonenzymatic method underwent relatively safe outcome, the extrapolation of data from any animal model to human valve implantation requires caution. Human applications will require additional longterm data because previous results of implantation of tissue-engineered porcine heart valves in pediatric patients have been catastrophic. ${ }^{18}$ Further studies will be required.

In conclusion, we demonstrate that xenogeneic porcine heart valves decellularized through a nonenzymatic process allow partial spontaneous autologous recolonization, with delayed and limited inflammation and calcifications. However, the recolonization process remains
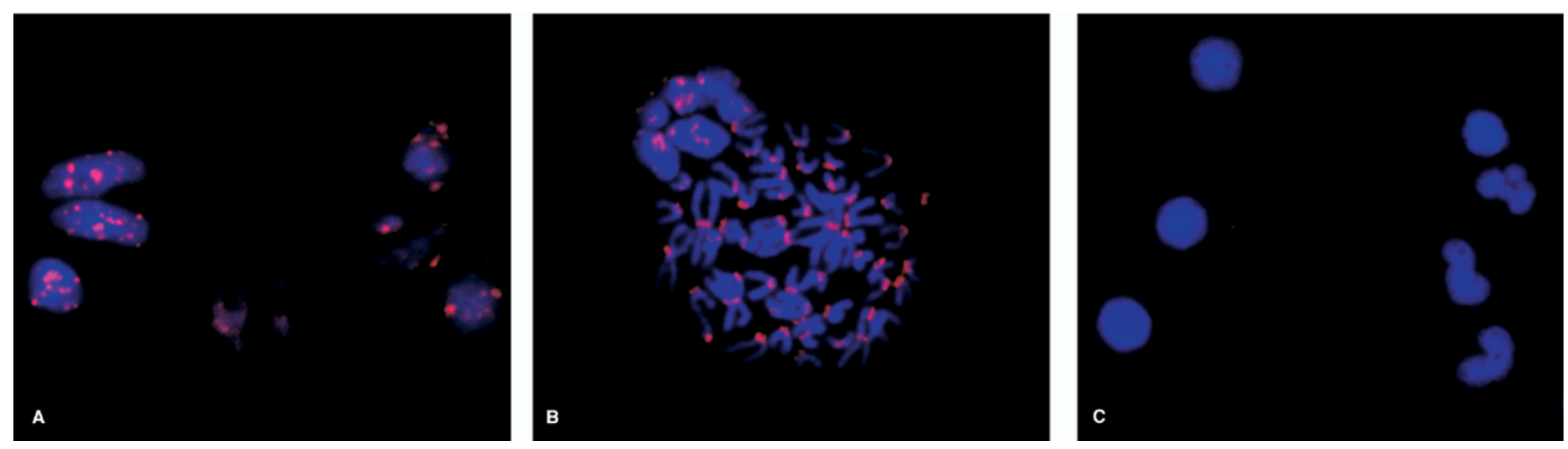

Figure 8. Results of fluorescence in situ hybridization on the cells eluted from the graft (A), a positive control on ovine blood (B), and a negative control on porcine blood (C). 
limited, suggesting that further improvement of the extracellular scaffold is mandatory before human implantation. In this setting G-CSF accelerated valve deterioration through increased inflammatory reaction and calcifications. Further studies shouldconsider alternative strategies to improve scaffold recolonization without inducing adverse effects on valve outcome.

We thank François Piumi, Joris Andrieux, Christophe Roumier, and Valérie Soenen for their assistance in developing the ovine molecular probe and Alexandre Eung for expert technical assistance.

\section{References}

1. Hammermeister KE, Sethi GK, Henderson WG, Oprian C, Kim T, Rahimtoola S. A comparison of outcomes in men 11 years after heart-valve replacement with a mechanical valve or bioprosthesis. Veterans Affairs Cooperative Study on Valvular Heart Disease. $N$ Engl J Med. 1993;328:1289-96.

2. Shinoka T, Breuer CK, Tanel RE, Zund G, Miura T, Ma PX, et al. Tissue engineering heart valves: valve leaflet replacement study in a lamb model. Ann Thorac Surg. 1995;60(suppl):S513-6.

3. Hoerstrup SP, Sodian R, Daebritz S, Wang J, Bacha EA, Martin DP, et al. Functional living trileaflet heart valves grown in vitro. Circulation. 2000;102(suppl 3):III44-9.

4. Steinhoff G, Stock U, Karim N, Mertsching H, Timke A, Meliss RR, et al. Tissue engineering of pulmonary heart valves on allogenic acellular matrix conduits: in vivo restoration of valve tissue. Circulation. 2000;102(suppl 3):III50-5.

5. Wilcox HE, Korossis SA, Booth C, Watterson KG, Kearney JN, Fisher J, et al. Biocompatibility and recellularization potential of an acellular porcine heart valve matrix. J Heart Valve Dis. 2005;14: 228-37.

6. Stock UA, Nagashima M, Khalil PN, Nollert GD, Herden T, Sperling JS, et al. Tissue-engineered valved conduits in the pulmonary circulation. J Thorac Cardiovasc Surg. 2000;119:732-40.

7. Orlic D, Kajstura J, Chimenti S, Jakoniuk I, Anderson SM, Li B, et al. Bone marrow cells regenerate infarcted myocardium. Nature. 2001; 410:701-5.

8. Perry TE, Kaushal S, Sutherland FW, Guleserian KJ, Bischoff J, Sacks M, et al. Thoracic Surgery Directors Association Award. Bone marrow as a cell source for tissue engineering heart valves. Ann Thorac Surg. 2003;75:761-7.

9. Hoerstrup SP, Kadner A, Melnitchouk S, Trojan A, Eid K, Tracy J, et al. Tissue engineering of functional trileaflet heart valves from human marrow stromal cells. Circulation. 2002;106(suppl 1):I14350 .

10. Minamino K, Adachi Y, Okigaki M, Ito H, Togawa Y, Fujita K, et al. Macrophage colony-stimulating factor (M-CSF), as well as granulocyte colony-stimulating factor (G-CSF), accelerates neovascularization. Stem Cells. 2005;23:347-54.

11. Kawada H, Fujita J, Kinjo K, Matsuzaki Y, Tsuma M, Miyatake H, et al. Nonhematopoietic mesenchymal stem cells can be mobilized and differentiate into cardiomyocytes after myocardial infarction. Blood. 2004;104:3581-7.

12. Booth C, Korossis SA, Wilcox HE, Watterson KG, Kearney JN, Fisher $\mathrm{J}$, et al. Tissue engineering of cardiac valve prostheses I: development and histological characterization of an acellular porcine scaffold. J Heart Valve Dis. 2002;11:457-62.

13. Korossis SA, Booth C, Wilcox HE, Watterson KG, Kearney JN, Fisher $\mathrm{J}$, et al. Tissue engineering of cardiac valve prostheses II: biomechanical characterization of decellularized porcine aortic heart valves. J Heart Valve Dis. 2002;11:463-71.
14. Gulbins H, Goldemund A, Anderson I, Haas U, Uhlig A, Meiser B, et al. Preseeding with autologous fibroblasts improves endothelialization of glutaraldehyde-fixed porcine aortic valves. J Thorac Cardiovasc Surg. 2003;125:592-601.

15. Rieder E, Kasimir MT, Silberhumer G, Seebacher G, Wolner E, Simon $\mathrm{P}$, et al. Decellularization protocols of porcine heart valves differ importantly in efficiency of cell removal and susceptibility of the matrix to recellularization with human vascular cells. J Thorac Cardiovasc Surg. 2004;127:399-405.

16. Rieder E, Seebacher G, Kasimir MT, Eichmair E, Winter B, Dekan B, et al. Tissue engineering of heart valves: decellularized porcine and human valve scaffolds differ importantly in residual potential to attract monocytic cells. Circulation. 2005;111:2792-7.

17. O'Brien MF, Goldstein S, Walsh S, Black KS, Elkins R, Clarke D. The SynerGraft valve: a new acellular (nonglutaraldehyde-fixed) tissue heart valve for autologous recellularization first experimental studies before clinical implantation. Semin Thorac Cardiovasc Surg. 1999; 11(suppl 1):194-200.

18. Simon P, Kasimir MT, Seebacher G, Weigel G, Ullrich R, SalzerMuhar U, et al. Early failure of the tissue engineered porcine heart valve SYNERGRAFT in pediatric patients. Eur J Cardiothorac Surg. 2003;23:1002-6.

19. Bechtel JF, Gellissen J, Erasmi AW, Petersen M, Hiob A, Stierle U, et al. Mid-term findings on echocardiography and computed tomography after RVOT-reconstruction: comparison of decellularized (SynerGraft) and conventional allografts. Eur J Cardiothorac Surg. 2005; 27:410-5.

20. Sayk F, Bos I, Schubert U, Wedel T, Sievers HH. Histopathologic findings in a novel decellularized pulmonary homograft: an autopsy study. Ann Thorac Surg. 2005;79:1755-8.

21. Goncalves AC, Griffiths LG, Anthony RV, Orton EC. Decellularization of bovine pericardium for tissue-engineering by targeted removal of xenoantigens. J Heart Valve Dis. 2005;14:212-7.

22. Orlic D, Kajstura J, Chimenti S, Limana F, Jakoniuk I, Quaini F, et al. Mobilized bone marrow cells repair the infarcted heart, improving function and survival. Proc Natl Acad Sci U S A. 2001;98:10344-9.

23. Norol F, Merlet P, Isnard R, Sebillon P, Bonnet N, Cailliot C, et al. Influence of mobilized stem cells on myocardial infarct repair in a nonhuman primate model. Blood. 2003;102:4361-8.

24. Iwanaga K, Takano H, Ohtsuka M, Hasegawa H, Zou Y, Qin Y, et al. Effects of G-CSF on cardiac remodeling after acute myocardial infarction in swine. Biochem Biophys Res Commun. 2004;325:1353-9.

25. Minatoguchi S, Takemura G, Chen XH, Wang N, Uno Y, Koda M, et al. Acceleration of the healing process and myocardial regeneration may be important as a mechanism of improvement of cardiac function and remodeling by postinfarction granulocyte colony-stimulating factor treatment. Circulation. 2004;109:2572-80.

26. Bhattacharya V, Shi Q, Ishida A, Sauvage LR, Hammond WP, Wu $\mathrm{MH}$. Administration of granulocyte colony-stimulating factor enhances endothelialization and microvessel formation in small-caliber synthetic vascular grafts. J Vasc Surg. 2000;32:116-23.

27. Powell TM, Paul JD, Hill JM, Thompson M, Benjamin M, Rodrigo M, et al. Granulocyte colony-stimulating factor mobilizes functional endothelial progenitor cells in patients with coronary artery disease. Arterioscler Thromb Vasc Biol. 2005;25:296-301.

28. Anderlini P, Przepiorka D, Champlin R, Korbling M. Biologic and clinical effects of granulocyte colony-stimulating factor in normal individuals. Blood. 1996;88:2819-25.

29. Noursadeghi M, Pepys MB, Gallimore R, Cohen J. Relationship of granulocyte colony stimulating factor with other acute phase reactants in man. Clin Exp Immunol. 2005;140:97-100.

30. Kang HJ, Kim HS, Zhang SY, Park KW, Cho HJ, Koo BK, et al. Effects of intracoronary infusion of peripheral blood stem-cells mobilised with granulocyte-colony stimulating factor on left ventricular systolic function and restenosis after coronary stenting in myocardial infarction: the MAGIC cell randomised clinical trial. Lancet. 2004; 363:751-6. 